\title{
Development of Quench Detection System for W7-X
}

\author{
Dietrich Birus $^{1}$, Thomas Rummel ${ }^{1}$, Marko Fricke ${ }^{1}$, Klaus Petry ${ }^{2}$, Horst Demattio ${ }^{2}$ \\ ${ }^{1}$ Max-Planck-Institut für Plasmaphysik, Euratom Association \\ Teilinstitut Greifswald, Wendelsteinstr. 1, D-17491 Greifswald, Germany \\ ${ }^{2}$ Forschungszentrum Karlsruhe, Institut für Prozessdatendatenverarbeitung und Elektronik, \\ Hermann-von-Helmholtz-Platz 1, D-76344 Eggenstein-Leopoldshafen, Germany
}

\begin{abstract}
The Quench Detection System of W7-X will consist of nearly 400 Quench Detection Units (QDU) for the fast and reliable supervision of the 70 superconducting coils and the 120 superconducting bus bar sections. There will be five control racks with about 80 QDU, a data acquisition unit, an AC-DC power supply with integrated DC-UPS unit in each of the racks and a PC based data management system as an overlay structure. Each QDU will have a special analogue input circuit realised as a programmable half bridge front end with different polarity-sensing and limiting functions for suppressing high dynamic voltages. Special filter design is included for noise-suppression and over voltage protection. A reconfigurable control/arithmetic unit offers possibilities of future expansions (e.g. all digital evaluation).

The QDU acquires and checks the differential voltages of the superconductors permanently. In case of a quench it triggers the fast discharge of the coils and the storage of the voltage signals on the memory unit. The quench signals are in the $\mathrm{mV}$ range and have to be clearly identified within a noisy and a high-voltage background within a few milliseconds.

Each QDU transfers the stored signal dates via a high-speed RS-485 serial interface with 20KV optical isolation barrier to an industrial type data acquisition unit. A second optically isolated RS-485-network enables interconnection of each QDU in the control rack (Compound-Mode of QDU).

The QDU are designed with an internal failsafe, programmable self test and redundancy feature, broken wire check of the quench detection cables and connectors inside and outside of the cryostat of W7-X.

All QDU will be fed via an UPS supported 24V DC bus through a high voltage isolated DC-DC transformer on each unit.

The design of the Quench Detection System allows operation under high voltage levels of up to 8kV and under magnetic stray field levels up to 30mT. The front end is very well isolated and the outputs of the QDU are strictly separated by optical isolating transformers. The differential inputs are equipped with 500V short-time over voltage protection.

The paper will give an overview of the design of the first prototype.
\end{abstract}

\section{Quench Detection System}

The Stellerator W7-X has a superconducting magnet system. The zero resistance superconducting state of the superconducting coils must be supervised permanently. In case of partly or complete loss of the superconducting state of any coil or of any of the bus bar sections, the magnet system must be discharged rapidly and the power supplies of the magnet system must be turned off.

The Quench Detection System have to be the ability to suppress the whole RF and LF noise and voltages induced by the DC magnet stray field of the Stellerator system, which surround the magnet system. It must withstand the dynamic high voltages, which occur during discharging the magnet system. It means that the design of the measuring part must be galvanic ally isolated from the power supply of the Quench Detection System as well as from the control system of the magnet system power supply. The configuration control of the Quench Detection Unit and the data transfer from the Quench Detection Unit to the Real Time Data Acquisition Unit must be galvanic ally isolated too. 
For 70 superconducting coils and more than 120 superconducting bus bar sections 360 Quench Detection Units are needed. The Quench Detection Units will be mounted in five control cabinets; it means five Quench Detection Sub Systems. Each cabinet contains 72 Quench Detection Units, one real time Data Acquisition Unit and a 24V DC power supply with integrated DC UPS. The Data Acquisition Unit communicates with each of the Quench Detection Units via two high speed RS 485 interfaces. It allows to configure the Quench Detection Units and the data transfer from the Quench Detection Unit to the Data Acquisition Unit. Each of the data acquisition units is connected with the neighbouring units by an Ethernet Bus and via a fibre optic. The first of the Quench Detection Subsystems have an Ethernet fibre optic connection to the Quench Detection Host System for supervising and configuration of the Quench Detection Units and subsystems (Fig. 1).

The measuring part and detecting part of each Quench Detection Unit is connected to the electrical potential of the superconducting coils through the quench cable. The signal acquisition and the analogue and digital signal processing need to be performed at the high voltage level of the superconducting coils. The required galvanic isolation between the measuring and detecting part, the safety circuit and the control unit will be realised at the interfaces by high voltage optoelectronic couplers. For the DC supply of the whole Quench Detection Unit works a high frequency DC-DC converter with a high voltage isolation barrier. The required supply power at the $24 \mathrm{~V}$ DC input is up to $6 \mathrm{~W}$.

The DC supply of all these Quench Detection Units of one QD subsystem is realised by an AC-DC converter combined with a DC UPS, which works with a resonance circuit design. The 230V AC front end is strictly isolated from the $24 \mathrm{~V}$ DC output. The output delivers a nominal current up to 30A The DC UPS allows an independent supply of the whole subsystem for a time of up to 12 minutes. The circuit design of the AC-DC converter will ensure the effective suppression of voltage spikes and harmonics from the mains. There are only $60 \mathrm{kHz}$ ripples and about $10 \mathrm{mV}$ RMS at the $24 \mathrm{~V}$ DC output, but no low frequency distortion and no high frequency peaks above over $1 \mathrm{mV}$.

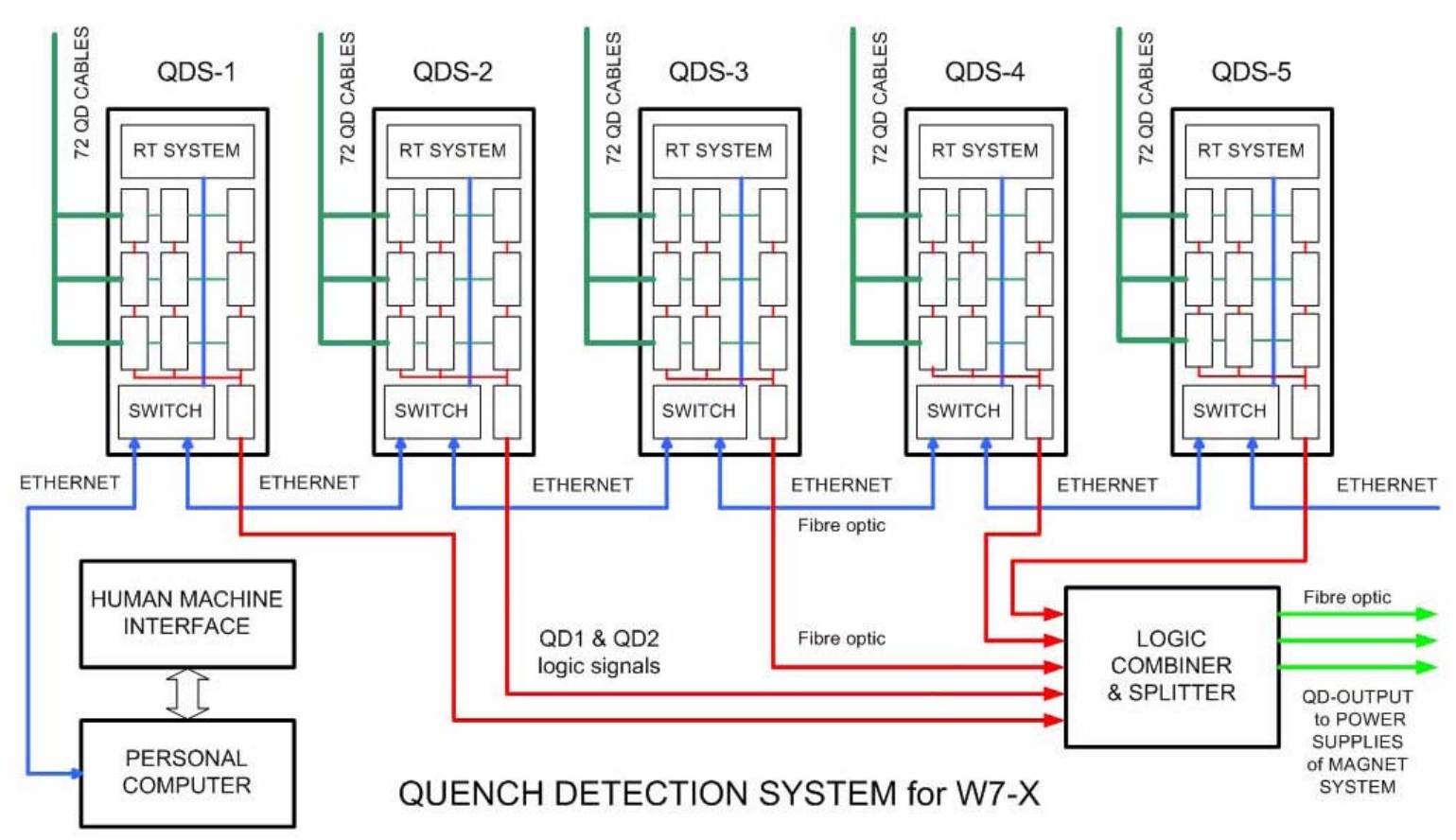

Fig. 1 Quench Detection System of W7-X 
The interconnection of the interfaces of the Quench Detection Subsystems and the Human Machine Interface is realised by an optical fibre bus with Ethernet structure. Each of the data acquisition units has an electric Ethernet port, whose is connected to an Ethernet switch in the subsystem. This switch has two electrical ports, one for the data acquisition unit and one for the free connection to a laptop as well as two fibre optic ports to connect the two fibre optic Ethernet bus lines. This system is expandable for up to 100 bus subscribers.

The two logic state signals QD1 and QD2 are delivered as electric signals in relation to the internal ground of the subsystem. The signals will be transferred to a fibre optic state signal by electric optic converters in each subsystem. A combiner resumes the state signals of all the Quench Detection Subsystems and is followed by a splitter, which divides the state signals to the three control units of the magnet system power supplies. There is a strict separation between the Quench Detection Subsystems and the magnet system power supplies.

All subsystems and components will be successfully tested for EMC, in accordance with DIN EN 55022 for electromagnetic emission and DIN EN 55024 for electromagnetic noise immunity. The units also passed have to pass a DC magnetic field immunity test at $30 \mathrm{mT}$ and a high voltage isolation test at $17 \mathrm{kV}$.

\section{Design of Quench Detection Unit}

Each QDU will have a special analogue input circuit which is realised as a programmable half bridge front end with different polarity-sensing and limiting functions for suppressing high dynamic voltages. Special filter design is included for noise-suppression and over voltage protection.

A reconfigurable control/arithmetic unit offers the possibility for future expansions (e.g. all digital evaluation). The QDU acquires and checks the differential voltages of the superconductors permanently. In case of a quench it triggers the fast discharge of the coils and stores the voltage signals in the memory unit.

The quench signals are in the $\mathrm{mV}$ range and have to be clearly identified within a noisy and a highvoltage background within a few milliseconds. Each QDU transfers the stored signal dates via a highspeed RS-485 serial interface with $20 \mathrm{KV}$ optical isolation barrier to the industrial type data acquisition unit.

A second optically isolated RS-485-network enables interconnection of each QDU in the control rack (Compound-Mode of QDU). The QDU are designed with an internal failsafe, programmable self test and redundancy feature, broken wire check of the quench detection cables and connectors inside and outside of the cryostat of W7-X.

Each QDU will be powered via an UPS supported 24V DC bus through a high voltage isolated DCDC transformer.

\section{Detailed description of Quench-Detector Unit UNIQD (Fig. 2)}

The Universal Quench Detector Unit “UNIQD” is capable of different application modes:

- Two superconducting Coil-part Half-Bridge mode with adjustable balancing

- Single superconducting Coil / Compensation wire Half-Bridge mode with adjustable balancing

- Superconducting Bus Bar detection mode (Single-Detection mode) 
There are two operational detector modes:

- $\quad$ independent 2-way mode (separately evaluated QD-Signals for e.g. experimental purposes)

- one-way-mode with fully redundancy capability and automatic onboard circuit check.

Mode 2 is the standard operation mode. In this mode the separate QD-outputs are simultaneously triggered by the detector.

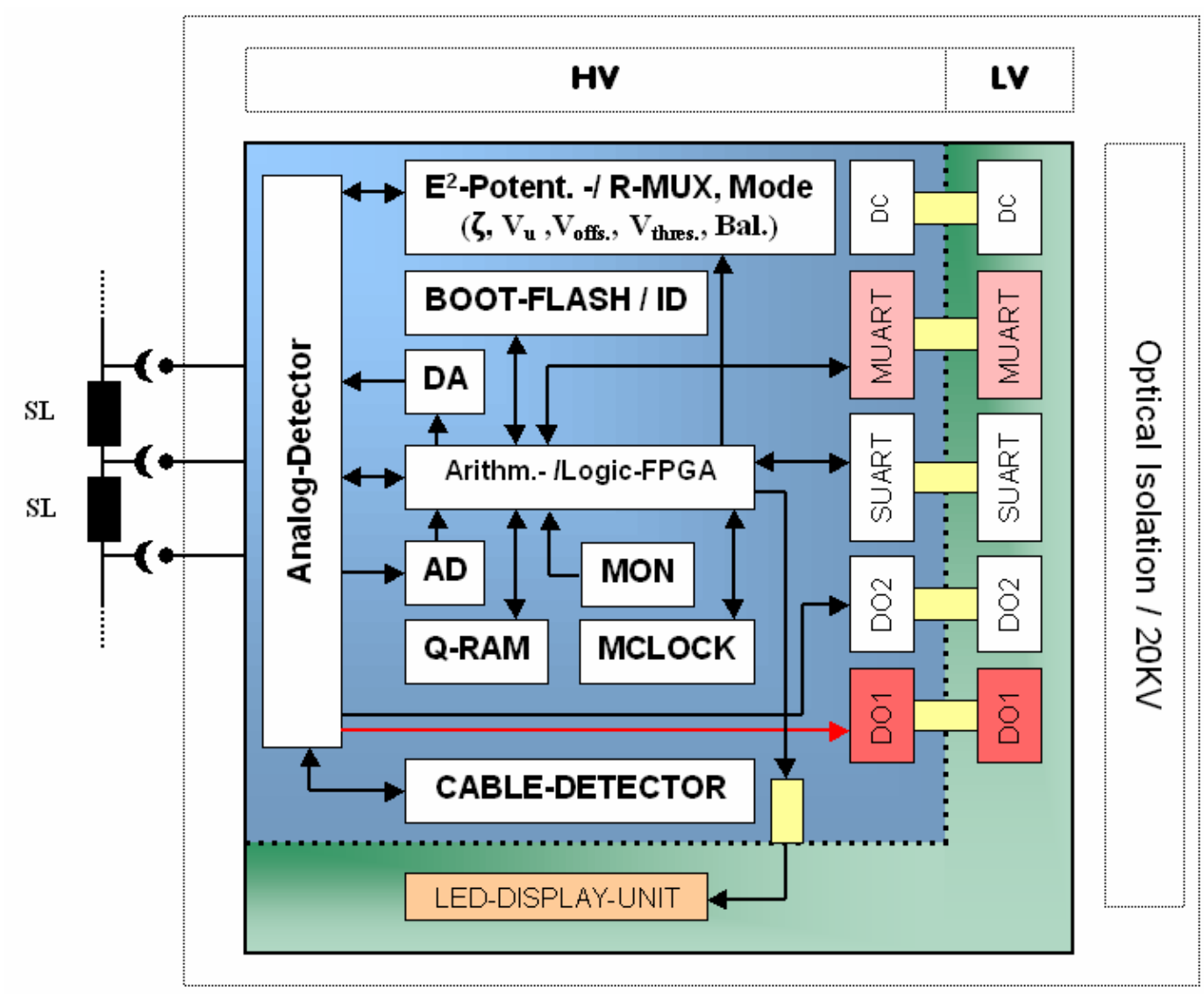

Fig. 2 Simplified schematic of universal Quench Detector UNIQD

A special feature of the UNIQD is the possibility of balancing coil-parts in the range of 0 to $100 \%$ by digital programmable differential input divider network. Together with the polarity sensing detector circuit for blanking reverse voltage a maximum of noise immunity should be obtained.

High-voltage input circuit is based on a well defined cascaded network, consisting of combinations of fast arresters, bi-directional transient suppresser components and further low-cap drain techniques. No varistors are used in this design to keep thermal input impedance changes at a minimum. The input is protected against transient voltages of up to $500 \mathrm{~V}$ (single ended) and up to $1000 \mathrm{~V}$ (differential). HVprotection to earth-potential is done by special board layout and digital onboard interfaces by fast $20 \mathrm{kV}$ fibre optics. Overall protection to earth is realised at a minimum of $17 \mathrm{kV}$.

Accurate detection of the quench is achieved by high quality instrumental amplifier techniques combined with precision low offset, low temperature drift comparators. Digital potentiometers for adjusting balance, threshold and calibration have an initial accuracy of better than $0.3 \%$ with a resolution of 8 Bit each. Tolerance-spreading of each QD-Unit can be compensated by programmable calibration of input amplifiers. Therefore PnP-usage and replace ability of different QD-Units is ensured. 
The detector itself consists of:

- 2 x 2 independent digital adjustable analogue threshold detectors (precision bipolar 2-way detector)

- 2 low tolerance adjustable filter networks

- 12 Bit-Sampling unit (10sec onboard ring buffer at 100ks/sec)

- Multifunctional digital supervisor circuit (watchdog, reset, power, temperature and state monitoring)

- Programmable digital detector (selectable analogue and /or digital evaluation)

- Regulated Charge-pump power supply for reference and analogue circuit power of detector (high immunity to magnetic stray field)

- 2 x High-Speed / 20kV High-Voltage protected 2.5Mbit RS485-Interfaces (max. 10Mbit)

- 2 x 20kV High-Voltage protected 24V QD-Outputs

- Multiple Onboard parameter retention memory (EEPROM)

- Fully remote reconfigurable system via RS485-interface (digital part)

- Fail-Safe design (supervised Power-Up, Online System-Check, Redundancy detector circuit; internal fail-safe electronic construction; switch able cable-detection)

The following list summarises the main technical data of the UNIQD:

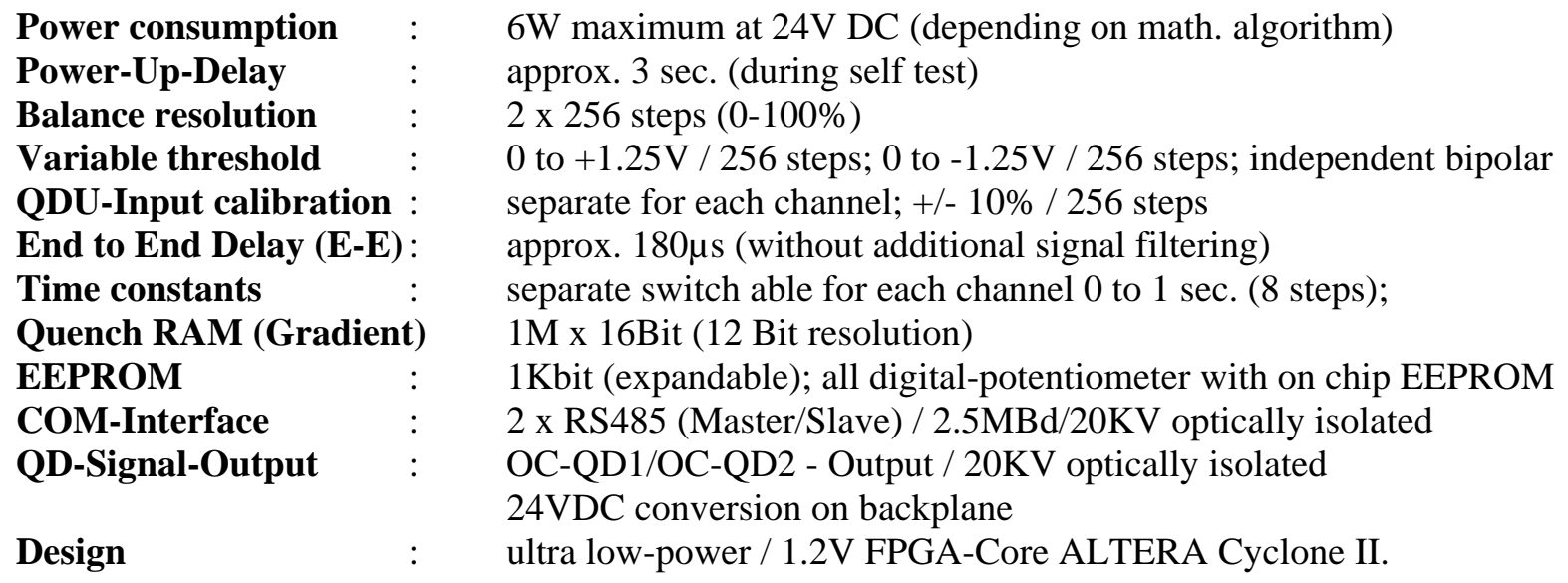

\section{Design of Quench Detection Backplane}

The backplanes serve as connector for 8 QD-Units to the RT-Data Acquisition-System and provides designated "OR" logic of all QD-signals in a control cabinet (9 backplanes, 72 QDU). The resulting 24VDC-QD-signal is fed through a high voltage optoelectronic coupler to the logic combiner \& splitter in W7-X.

Each backplane features its own power-supply, which is used for industrial signal conversion and monitoring functions at the front-panel of the QD-Units (Split Power System in the QD-Unit by HV/LV-potential). For evaluation purposes additional standard RS232 interfaces are provided that offer simple connection of first QD-Unit to a Laptop-Computer without RS485 interface.

RS485 network electronics are an integral part of the backplane. Different impedance-matching for High-Speed communicating via RS485 is not possible in the QD-unit, because the HV-potential inside the QD-Unit requires complete sealing of the electronics with special HV silicon compound.

The backplane has a similar layout as the QD-unit with integrated shielding layers for optimized EMC-performance. 


\section{Design of Data Acquisition Unit}

The data acquisition is carried out with a robust, industry standard real-time system of Jaeger Messtechnik GmbH. The real time (RT) system is connected via a fast RS 485 bus to the Quench Detection Unit (QDU) for its configuration and parameterisation. A controlling PC serves for initializing the RT system and for displaying the recorded quench development. Separate lines trigger the RT system in case of a quench. All quench events are associated with a time stamp; a broadcast signal synchronizes the memory for quench events on the QDU. The time resolution for successive quench events is $25 \mu$ s. It is possible to group QDUs via the RT system to analyse coupling effects between individual coils if required.

The main components of the RT system are:

- ADSP21162 80MHz board with boot loader option and Ethernet interface

- 3 X card with 32 optical isolated digital inputs

- transistor output card with 16 outputs

- memory card PCMCIA PC CARD ATA 128MB

- $\quad$ interface card with 4 RS-485 ports

The 19“system (see figure 3) is built-up by independent modules and is extendable on demand.

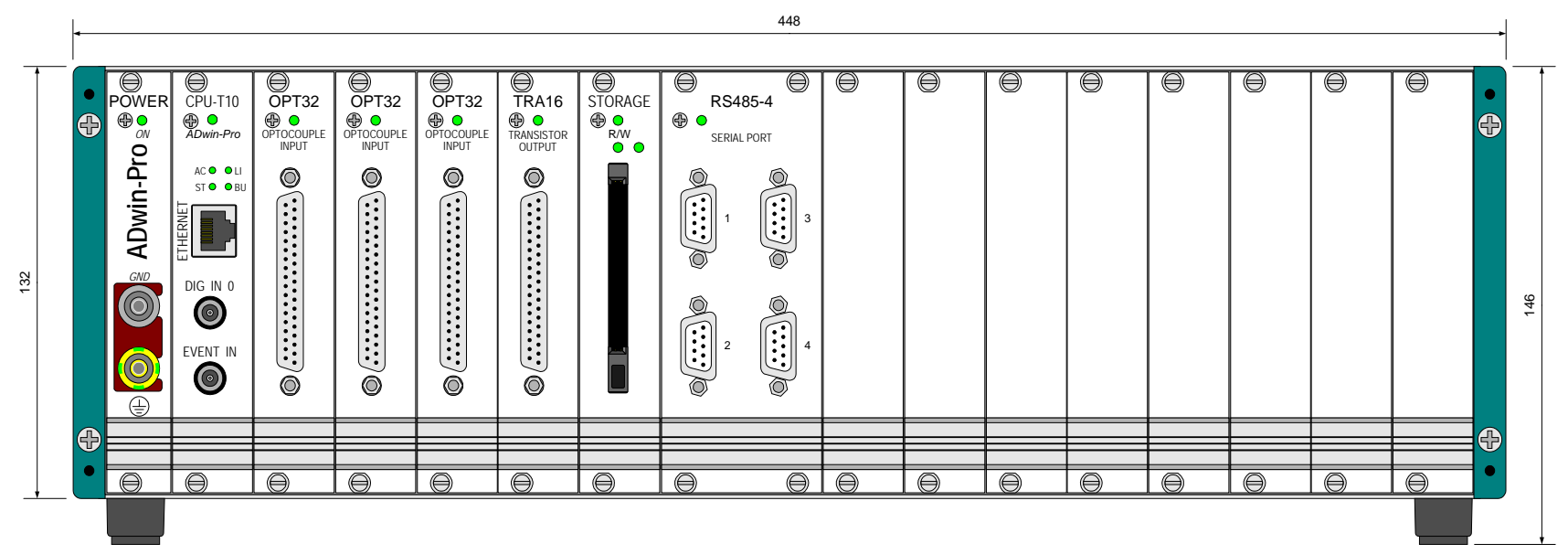

Fig. 3 Scheme of the real-time system in its basic configuration; there are 8 slots left for expansion modules.

\section{Summary}

The description of the quench detection system development demonstrates the new open concept, based on an expandable design of all units. There are some new ideas in realising a strictly electrical separation of all system units, where the interfaces isolation between the measuring and detection units and the real time signal processing system must withstand nearly $20 \mathrm{kV}$. The suppression of the RF and LF noise and the dynamic range of the quench detection unit have a very high performance based on filter technique and decoupling networks. The parameters of the digital part of the Quench Detection Unit are fully remote configurable via the high speed RS485 interface and the design of the unit is made complete fail-safe. The whole design of the Quench Detection System is realised to withstand a magnetic DC field up to $30 \mathrm{mT}$ in all operation modes. 


\section{References}

M. Borlein: Optimierung eines Quench-Detektions-Systems für supraleitende Magnetspulen Diplomarbeit; Forschungszentrum Karlsruhe; Institut für Technische Physik, 2004

Jäger GmbH: ADwin Pro Messwerterfassungssystem; Produktbeschreibung; Lorsch, 2006 www.adwin.de

ELMIC GmbH: Einkanal-Hochspannungs-Optokoppler, EL-HV-50; Produktbeschreibung; Duisburg 2006; www.elmic.de

Bürger Elektronik GmbH: Hochspannungsisolierter DC-DC-Wandler; Produktbeschreibung; Reutlingen 2006; www.buerger-elektronik.de

Altera Corp.: $\quad$ Cyclone II FPGA, Data Sheet; San Jose, CA, USA, 2006; www.altera.com

Analog Devices: Analog Digital Converters, Data Sheets; Norwood, MA, USA, 2006; www.analog.com

Burr Brown: Instrumentation Amplifiers, Data Sheets; Phoenix, AZ, USA, 2006; www.burrbrown.com

Maxim IC: $\quad$ Drivers, Converters; Data Sheets; Sunnyvale, CA, USA; 2006; www.maxim-ic.com 